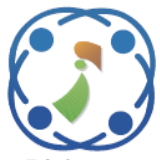

\title{
Pixel Classification Based on Local Gray Level Rectangle Window Sampling for Amniotic Fluid Segmentation
}

\author{
Putu Desiana Wulaning Ayu ${ }^{1,2} \quad$ Sri Hartati* ${ }^{3 *}$ \\ ${ }^{I}$ Doctoral Program Department of Computer Science and Electronics, Faculty of Mathematics and Natural \\ Science, Universitas Gadjah Mada, Yogyakarta, Indonesia \\ ${ }^{2}$ Department of Information Technology, Faculty Computer and Informatics, Institut Teknologi dan Bisnis STIKOM \\ Bali, Bali, Indonesia \\ ${ }^{3}$ Department of Computer Science and Electronics, Faculty of Mathematics and Natural Science, Universitas \\ Gadjah Mada, Yogyakarta, Indonesia \\ * Corresponding author’s Email: shartati@ugm.ac.id
}

\begin{abstract}
This study analyses the use of a pixel classification model to segment amniotic fluid areas on ultrasound (US) images characterized by noise, blurry edge, artifacts, and low contrast. In contrast with the previous methods, this study constrains a training set of pixels based on neighbourhood information with the rectangle window sampling method used to determine the characteristics of each pixel in its environment specifically. The feature extraction is no longer based on the global characteristics of the object rather by taking the value of each pixel in the object area using the sampling window. This research also combines the local first-order statistical methods and gray level information in the window to obtain the characteristics of each pixel. Furthermore, Random Forest and Decision Tree (C.45) were used to classify each pixel into four classes, namely amniotic fluid, placenta, uterus, and fetal body. The classification performance testing of pixel sampling data showed that the Random forest with $5 \times 7$ window sizes achieved the highest performance at $99.5 \%$ accuracy, precision, and recall, respectively. Furthermore, the proposed model was evaluated using 50 new test US images to segment the amniotic fluid area. According to experimental result, proposed models can produce better segmentation area with an increase in the IoU value by $18.3 \%$ or a Jaccard coefficient value rate of 0.183 in the range of $0-1$ with the previous state of the art method. Furthermore, the proposed model reduces the error rate and improves accuracy by $6.61 \%$ and $84.77 \%$, respectively.
\end{abstract}

Keywords: Amniotic fluid, Pixel classification, Window sampling, Local first-order statistical method, Gray level feature.

\section{Introduction}

Ultrasound imaging is used to visualize the internal body structure, such as soft tissue, tendons, joints, blood vessels, muscles, and organs, through diagnoses and interventional procedures [1]. It is also used by doctors to determine the volume of amniotic fluid in pregnant women, which functions to protect the fetus in the event of a collision in the uterine wall, thereby protecting the umbilical cord [2]. Deficiency in the amount of amniotic fluid, also known as Oligohydramnios, affects fetal movement, thereby leading to deformation and possible death.
Meanwhile, an excess amount of this fluid (polyhydramnios) leads to pre-mature labor, fetal macrosomia, fetal malposition, and increased risk of pregnancy disorders [3]. Therefore, in order to detect these conditions, the doctor uses the Single Deep Pocket (SDP) [4], to measure the vertical dimensions or perpendicular pull in the largest uterine quadrant or Maxima Vertical Pocket of pregnant women [5]. The initial stage of the SDP method is used to determine the deepest side of the area. It is considered an amniotic fluid, which depends on the knowledge and experience of a doctor and requires time and accuracy. This is because the area comprises of several body parts of the fetus, such as umbilical cord, 
placenta, uterus, and a less strict border between amniotic fluid and other parts.

Therefore, the segmentation method is performed in order to obtain the amniotic fluid area on the Bmode ultrasound image, which has noise, blurry edge, artifact, and low contrast qualities [6]. Ultrasound images have relatively low contrast with lots of noise around the object or organ, which needs to be observed. The effect of sound reflection on the object also causes an unexpected shadow capable of complicating object identification. Furthermore, the boundaries between the organs are not clearly visible, thereby causing blurry edges effect, which requires a segmentation method to identify each character of the object in more detail by recognizing the pattern between each and the surrounding pixels. The Bmode ultrasonography is generally a grayscale image with each pixel having its own gray level intensity. Yan et al. [7] researched the detection of amniotic fluid areas and fetal tissues in fetal ultrasound (US) images using the Encoding-Decoding CNN method. The proposed model performance in class specified evaluation had a $78.16 \%$ accuracy and $54.6 \%$ IoU for the amniotic fluid class. In addition, studies carried out on pixel classification model approaches by Qian et al. [8], Pazinato et al. [9], and Rosati et al. [10] for atherosclerotic carotid plaque, ultrasound tissue images and carotid wall segmentation on US images, showed relatively satisfying results.

This study proposes a segmentation model that classifies each pixel of the input image based on the neighbouring pixel information by using a sampling window approach that takes and processes the gray level values to its environment. The pixel classification scheme is carried out where the training set data is taken specifically for the neighbouring information for each pixel using a sliding rectangle window. The proposed pixel classification model consists of three stages. The first collects the pixel datasets using several size rectangles windows of $3 \times 5$, $5 \times 7,5 \times 3,7 \times 57 \times 9$, and $9 \times 7$ by selecting the sampling area at the core and border areas. The second stage performs feature extraction using first-order statistical based on local information window. The pixel value information in this window is further processed using local statistical first order feature extraction which changed because the concept was used to carry out sampling based on certain window and not on a single intact area of the object that had been previously conducted. The third stage classifies each pixel using the Random Forest and Decision Tree methods to obtain the amniotic fluid area. The performance of the two classifiers is seen from the Accuracy, Precision, and Recall values of each class. Finally, the proposed model's performance was determined by comparing the segmentation image with the ground truth using the Dice Similarity Coefficient (DSC) and Error Rate (ER). The contributions of this research are as follows:

1. Constructs a training set of pixels based on proximity information using the rectangle window sampling method. It specifically sees the characteristics of each pixel against the environment, provides more accurate segmentation results, especially in amniotic fluid boundary areas that tend to be indistinct.

2. Extracts feature values based on window sampling using the local first-order statistical method, which consists of variance, standard deviation, contrast, skewness, mean, entropy, and kurtosis. Furthermore, the gray level based on the window sampling feature is also added to strengthen each pixel's characteristics in the amniotic fluid.

The remaining part of this research is organized as follows. Section II explains some related work on the pixel classification models using medical images, while section III provides a more detailed description of the proposed method. Section IV contains experimental and result in an analysis of the proposed method, and section $\mathrm{V}$ concludes the research.

\section{Related work}

There is an increase in the development of the pixelwise classification method on the B-mode ultrasound image segmentation in recent years due to its ability to improve performance [11]. Yin et al. (2020) carried out kidney segmentation using subsequent boundary distance regression and pixel classification networks to segment the kidneys automatically [11]. However, there are still some challenges faced, namely the US images could be corrupted by shady or blurry artifacts, which could affect the image segmentation accuracy.

The pixelwise classification model is also used to segment spinal ultrasound images to obtain bone surfaces and active shadows in vertebrates [12]. The initial process in the research conducted by Berton et al. was used to create a training data set by taking random pixels from the existing vertebrate image in the datasets. Furthermore, feature extraction was carried out using gradient, phase symmetry, and bone probability map, which was classified using the Random Forest method with an accuracy of $91.1 \%$ for the active shadow class and $81.97 \%$ for the bone surface. Because it uses a very large number of features, the computation time of this proposed model is the next challenge because it still requires a relatively high computation time of 24 seconds per image. 


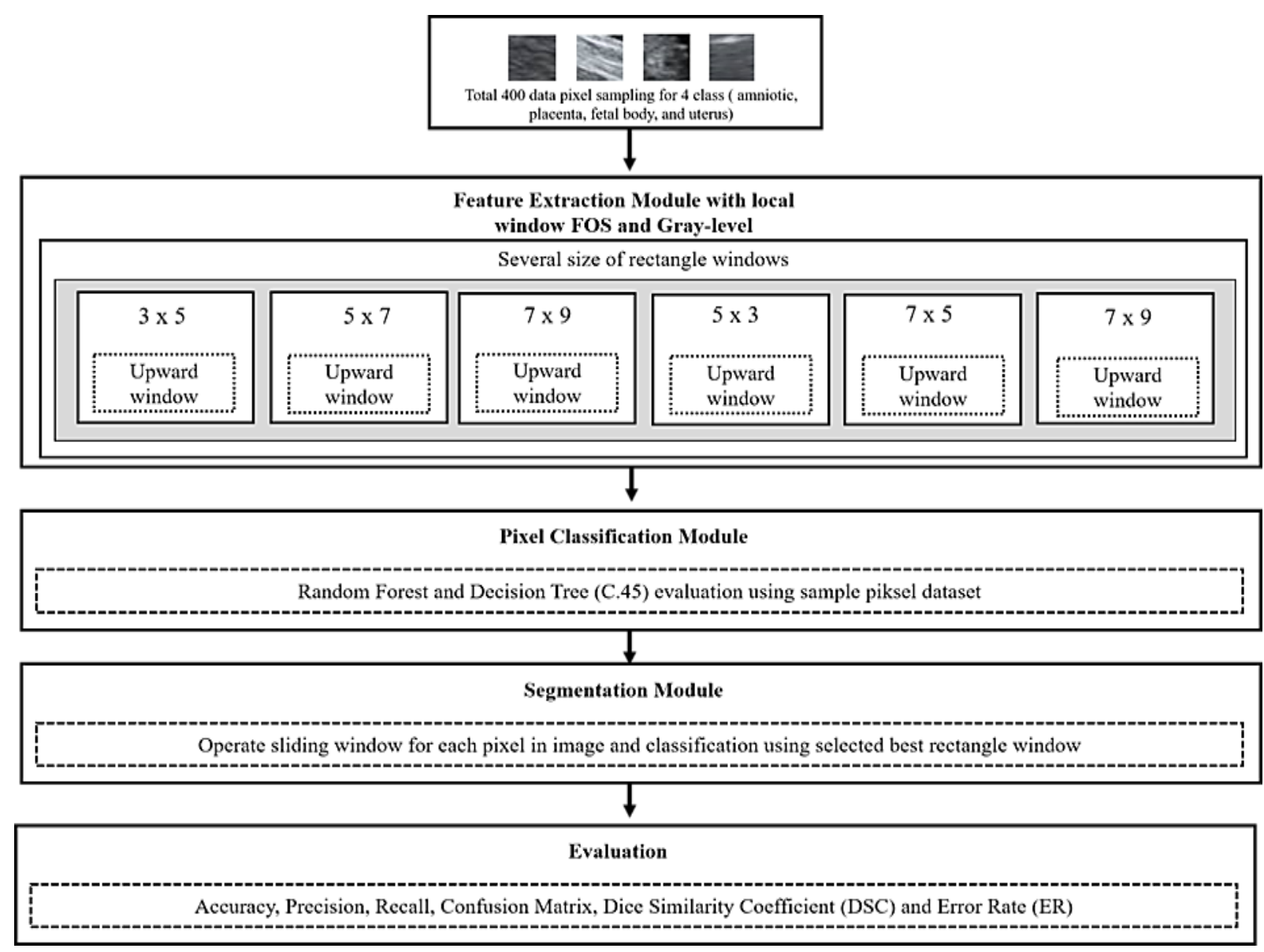

Figure. 1 Flow model of pixel classification

Meanwhile, the Intravascular Ultrasound (IVUS) images implemented the pixelwise classification method to classify pixels based on texture features using the ensemble Support Vector Machine [13]. A texture-enhanced deformable model (TEDM) is proposed to accurately detect these borders by incorporating texture information with the morphological factors of deformable model. There are several limitations mentioned in the research, including this method is not fully automatic because it requires manual labels in one IVUS training image and need to pre create training database that is large enough to account for all the different appearances of IVUS images

Several studies were carried out to determine the use of window size in sampling models for pixelwise classification. For instance, Qian et al. [8] utilized a window size of $9 \times 9$ to extract gray level value features as a sampling model. Auto-context iterative algorithm were employed to effectively integrate features from ultrasound images and later also the iteratively estimated and refined probability maps together with pixel-wise classification methods. Pazinato et al. [9] investigated the combined use of 9 $\times 9,11 \times 11$, and $13 \times 13$ window sizes in the Statistical Moment feature. The results showed that the $13 \times 13$ window was better than the $9 \times 9$ and 11 $\times 11$ windows, which explains that with a larger window size of the neighbourhood pixel information is available. In this study stated that however, that it is important to limit the RoI size to a maximum in order not to break the pixel's spatial coherence assumption. Rosati et al. [10] randomly used 600 pixels drawn on 120 images to represent 4 classes, namely adventitia, intima-media complex, lumen, and noisy lumen. Furthermore, texture features such as GLCM, Spatial Gray Level Dependence Matrix, and Gray Level Difference Matrix were drawn based on the area of specified window size $7 \times 15,15 \times 7$, $7 \times 5$ and $5 \times 7$. The Feed-Forward Neural Networks (FFNNs) classification method was used to classify each pixel into 4 classes at $79.5 \%$ accuracy, $87 \%$ sensitivity, and $72 \%$ specificity. Yan et al. [7] also carried out a research to detect the existing organs in the amniotic fluid to segment the amniotic area by using the CNN Encoding-Decoding approach where the input data is a region of each object. The results showed that the proposed method has an accuracy value of $78.16 \%$ and an IoU or Jaccard coefficient of $54.43 \%$ for detecting the amniotic fluid area. The formation of the dataset in this study is not specific to every object in the amniotic cavity. In addition, the 
training dataset is not formed from individual objects or organs but from one complete image in which there are several objects. This is likely to cause the IoU of amniotic fluid class to be relatively low.

From some previous literature related to the use of pixel wise classification on US images is able to produce satisfactory segmentation performance. In this study, the concept of pixel-wise classification is applied to the amniotic cavity object whose purpose is to segment the amniotic fluid area. There are several differences that are also proposed to answer the limitations of some of the previous work, namely the method of establishing a dataset using a local window sample in each object or organs and in addition to the observation window using a rectangle shape. The background in selecting the local sample window to form a dataset is that if the use of one whole images may contain important information that others could not capture, because operators of the ultra-sound machines might use different torque while capturing the images.

\section{Proposed method}

This study proposes a segmentation model with a window used to determine the blurry edges and pixel intensity diversity in amniotic fluid objects. This pixel classification model is used to observe a pixel pattern against its environment by using certain window size restrictions. In addition, the feature extraction method is carried out within the window's scope using First Order Statistical (FOS) features with a rectangle window used to perform observations in an upward direction and to trace the pixel scope, assuming it is in the boundary area. The pixel classification model in this study can be divided into five stages, as shown in Fig. 1. These include 1) pixel-level image feature using a rectangle sampling window, 2) feature extraction using first-order statistical methods based on the sample size, 3) features that have been extracted become input in the model training process uses the Random Forest and Decision Tree (C.45) as classifiers, 4) Amniotic fluid segmented with the trained classifier.

\subsection{Image acquisition}

The total dataset of B-mode ultrasonography images obtained in collaboration with Surya Husadha Hospital, Bali, is 60 images. The image data used as a dataset in this study is a single pregnancy with a susceptible age of 30-37 weeks. All images were taken from the Accuvix XG Machine and transducer with a frequency of $3.5 \mathrm{~Hz}$, lateral resolution corresponding from $3 \mathrm{~mm}$ to $0.2 \mathrm{~m}$, and in a JPG image format cropped to $427 \times 570$ pixels in size.
Cropping aims to eliminate patient information in the form of text and equalize the resolution of all image data, which is split into two parts, namely training (10 images) and testing (50 images). The study also obtained each ground truth image manually annotated from clinical experts.

\subsection{Constructing sampling pixel with rectangle window}

The formation of window sampling aims to take pixel samples from 4 areas or classes, namely the amniotic fluid, placenta, uterus, and fetal body, which are then processed to obtain feature values using the First Order Statistical (FOS) feature method. Furthermore, observations were made using several different window sizes with a rectangular shape. Each pixel point acts as the starting point of the observation, which forms an upward window from the starting point, as shown in Table 1 and Table 2. The pixel adjacency observation is then adjusted to the $n \times m$ size of the window. At the starting point of the pixel observation with coordinates $(x, y)$, the iteration of upward window formation is carried out by adding coordinates $x$ to the boundary $x+(n-1))$ where $\mathrm{n}$ denotes the row. After the observation is carried out

Table 1. Illustration of rectangle window formation size $3 \times 5$

$\mathbf{m}$

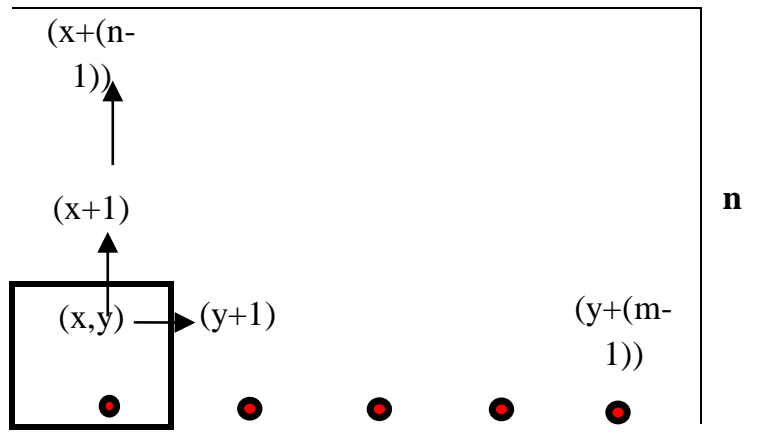

Table 2. Illustration of rectangle window formation size $5 \times 7$

m

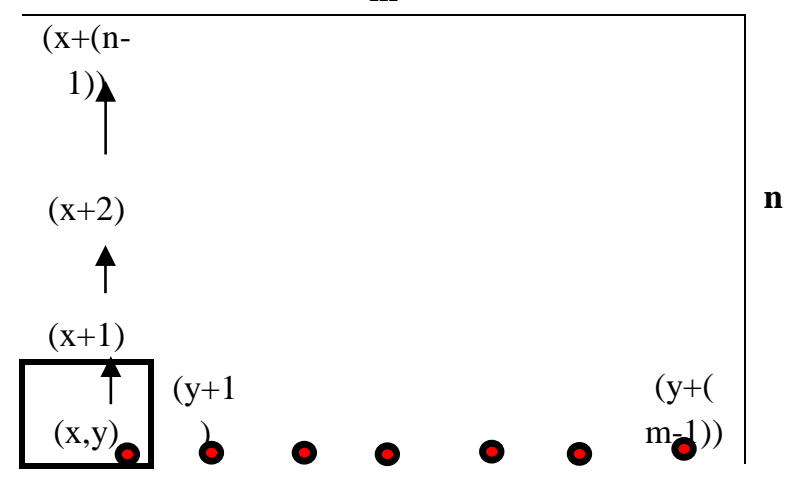


online and has reached the (n-1) line, the shifting coordinates $y$ by adding index $(y+1)$. Observation continues upward on pixel coordinates $(x, y+1)$ to line index $(n-1)$. This iteration stops when the $\mathrm{y}$ coordinates have increased by $(m-1)$, where $m$ is the number of columns of the window. As a result of this window formation, the gray-level intensity values of the pixels contained in the window are extracted using FOS.

\subsection{First order statistical feature by gray level local window}

Basically, the feature extraction using the FOS method analyses the distribution of gray level values on pixels in a region or object. In this study, the FOS extraction method was carried out using the scope of the window area. Each pixel is observed according to the window size; therefore, they possess varying areas. There are generally seven features used to describe the properties of image histogram and texture, namely mean, skewness, energy, kurtosis, standard deviation, variance, and entropy. The formula for calculating the feature values are shown in Eqs. (1)-(8).

Mean of local window:

$$
\bar{x}=\sum_{n} \sum_{m} \sum_{i=0}^{G-1} i_{(n, m)} P_{(n, m)}
$$

$\bar{x}$ is mean local window, where $P_{(n, m)}$ the probability density of intensity levels obtained by the following formula (2).

$$
P_{(n, m)}==\frac{h_{(i)}}{N}
$$

$n$ is the row of windows, $m$ is the column, $h_{(i)}$ represents the total number of pixels with the intensity level $(i), N$ is the total number of pixels in the window with $N=n \times m$.

Contrast local window:

$$
S^{2}=\sum_{n} \sum_{m} \sum_{i=0}^{G-1}\left(i_{(n, m)}-\bar{x}\right)^{2} P_{(n, m)}
$$

Where $G$ the maximum gray level inside the window. Skewness local window:

$$
\bar{x}_{3}=\sigma^{-3} \sum_{n} \sum_{m} \sum_{i=0}^{G-1}\left(i_{(n, m)}-\bar{x}\right)^{3} P_{(n, m)}
$$

Skewness is the degree of asymmetry of a distribution. Skewness is the third moment to the mean. Normal distributions (and other symmetrical distributions, for example the $t$ or Cauchy distribution) have a skewness of 0 (zero). Where $\sigma$ is standard deviation. Entropy local window:

$$
H=\sum_{n} \sum_{m} \sum_{i=0}^{G-1} P_{(n, m)} \log _{2}\left[P_{(n, m)}\right]
$$

Kurtosis local window:

$$
\bar{x}_{4}=\sigma^{-4} \sum_{n} \sum_{m} \sum_{i=0}^{G-1}\left(i_{(n, m)}-\bar{x}\right)^{4} P_{(n, m)}-3
$$

Kurtosis is the degree of tartness of a distribution, usually measured relative to the normal distribution. Kurtosis is calculated from the fourth moment to the mean. The normal distribution has kurtosis $=3$.

Standard deviation of local window:

$$
\sigma=\sqrt{\frac{1}{N-1} \sum_{n} \sum_{m}\left(x_{(n, m)}-\bar{x}\right)^{2}}
$$

Where $x_{(n, m)}$ is coordinate pixel $(n, m)$.

Variance of local window:

Variance is a measure of the diversity and distribution of data.

$$
\sigma^{2}=\frac{1}{N-1} \sum_{n} \sum_{m}\left(x_{(n, m)}-\bar{x}\right)^{2}
$$

\subsection{Dataset construction}

This section describes the pixel dataset characteristics used to train the classifier, which is formed from sampling pixels in each region of the amniotic cavity consisting of the fluid, placenta, uterus, and fetal body. Each pixel sample was taken using a rectangle window with sizes $3 \times 5,5 \times 3,5 \times 7$, $7 \times 5,7 \times 9$, and $9 \times 7$. In each region, 10 samples were taken using 10 different images culminating in 100pixel data windows. Therefore, each dataset contains a total of 400 instances consisting of 4 classes with varying dimensions of data in each window. The bigger the window size, the bigger the data dimension, as shown in Table 3.

\subsection{Training model with random forest}

Random forest (RF) is widely applied to solve some segmentation and classification problems in medical images [8,14-16]. RF classifier is a type of ensemble learning method used to build a final classifier with a set of collections from individual weak classifiers (M) such as the binary tree [16]. This study trained a random forest classifier to classify each pixel into the classes contained in the amniotic fluid, placenta, uterus, and fetal. This study uses 3 
Table 3. Data dimensions for each window size

\begin{tabular}{|c|c|c|c|}
\hline \multirow[b]{2}{*}{$\begin{array}{c}\text { Window } \\
\text { size }\end{array}$} & \multicolumn{3}{|c|}{ Feature } \\
\hline & $\begin{array}{l}\text { Gray } \\
\text { level }\end{array}$ & $\begin{array}{c}\text { First Order } \\
\text { Statistical } \\
\text { Feature by } \\
\text { Gray Level } \\
\text { Local Window }\end{array}$ & $\begin{array}{c}\text { Total } \\
\text { feature }\end{array}$ \\
\hline $3 \times 5$ & 15 & 7 & 22 \\
\hline $5 \times 3$ & 15 & 7 & 22 \\
\hline $5 \times 7$ & 35 & 7 & 42 \\
\hline $7 \times 5$ & 35 & 7 & 42 \\
\hline $7 \times 9$ & 63 & 7 & 70 \\
\hline $9 \times 7$ & 63 & 7 & 70 \\
\hline
\end{tabular}

important parameters in the RF, namely 20 depths of the tree, 2 samples per node, and 60 numbers of trees (Ntree) in the random forest, which is the optimal number to stabilize the out-of-bag-error in this study. The RF steps in this research are in accordance with previous studies [17], as follows:

Step 1: Randomly pick $k$ data points from the Training set.

Step 2: Build the decision tree associated with these $k$ data points.

Step 3: Choose the Number of Ntrees to build and repeat steps 1 and 2 .

Step 4: For a new data point, make each Ntrees trees to predict the category to which the data points belong, and assign the new data point in accordance with the category that wins the majority vote.

\section{Experimental result and analysis}

\subsection{Performance measure}

The parameters used to evaluate the classifier's performance in this study are Accuracy, Recall, and Precision as shown in Eqs. (9) - (11).

$$
\begin{gathered}
\text { Accuracy }=\frac{\mathrm{TP}+\mathrm{TN}}{\mathrm{TP}+\mathrm{FP}+\mathrm{TN}+\mathrm{FN}} \\
\text { Recall }=\frac{\mathrm{TP}}{\mathrm{TP}+\mathrm{FN}} \\
\text { Precision }=\frac{\mathrm{TP}}{\mathrm{TP}+\mathrm{FP}}
\end{gathered}
$$

$T P$ is True Positive (amniotic fluid pixel identified as amniotic fluid), FP is False Positive (non-amniotic fluid pixel as amniotic fluid), $T N$ is True Negative (non-amniotic fluid identified as non-amniotic fluid pixel), and $F N$ is False Negative (amniotic fluid pixel identified as non-amniotic fluid).

Furthermore, this study uses Dice's Coefficient (DSC), Error Rate (ER), and Jaccard index to evaluate the segmentation performance. DSC measures the similarity between two objects, which in the case of this study is the overlap computation of overlap area between the ground truth image and segmentation amniotic fluid area of the proposed model [16]. ER presents the ratio of misclassified image pixels over the total amount [18]. The DSC and ER formulas are shown in Eqs. (12) and (13).

\subsection{Performance result on data sampling pixel}

This section presents an evaluation of the amniotic fluid, placenta, uterus, and body fetal classification of each pixel sample that has been taken. The result of feature extraction at each window size becomes the input training in the classifier. The study also compares the performance of the Random Forest and Decision Tree (C.45) on each dataset with different window sizes using the accuracy, precision, recall, confusion matrix, and $k$-fold cross validation parameters. Cross-validation is a resampling procedure used to evaluate machine learning models on a limited data sample and avoid overfitting [19][20]. The procedure has a single parameter called $k$, which refers to the number of groups a given data sample is to be split. Therefore, the procedure is often called $k$-fold cross-validation, which forms a training set each time, one of the $k$ subsets is used while the other forms a $k-1$ subset while the average error across all $k$ trials is computed. The advantage of this method is that it matters less how the data gets divided. This is because every data point gets to be in a test set exactly once and $k-1$ time in a training set.

This study uses a $k$ value of 10 and comparison results of amniotic cavity organs to classify the window data in accuracy, precision randomly, and recall, as shown in Tables 4, 5, and 6, respectively.

The measurement in Tables 4, 5, and 6 showed that the observed window size of $5 \times 7$ produces higher precision, recall, and accuracy than others. Random forest with $5 \times 7$ window size achieved

Table 4. Accuracy of random forest for amniotic cavity organs classification

\begin{tabular}{|l|c|c|c|c|c|c|}
\hline \multirow{2}{*}{ Class } & \multicolumn{6}{|c|}{$\begin{array}{c}\text { Accuracy (\%) performance } \\
\text { comparison with several rectangle } \\
\text { window size }\end{array}$} \\
\cline { 2 - 7 } & $3 \times 5$ & $5 \times 3$ & $5 \times 7$ & $7 \times 5$ & $7 \times 9$ & $9 \times 7$ \\
\hline $\begin{array}{l}\text { Amniotic } \\
\text { Fluid }\end{array}$ & 98 & 92 & 99 & 99 & 95 & 87 \\
\hline Placenta & 92 & 89 & 100 & 90 & 89 & 96 \\
\hline Uterus & 99 & 88 & 100 & 88 & 89 & 97 \\
\hline $\begin{array}{l}\text { Fetal } \\
\text { Body }\end{array}$ & 86 & 98 & 99 & 100 & 100 & 87 \\
\hline $\begin{array}{l}\text { Average } \\
\text { Accurac } \\
\text { y }\end{array}$ & 93.7 & 91.7 & $\mathbf{9 9 . 5}$ & 94.2 & 93. & 91.7 \\
\hline
\end{tabular}


Table 5. Precision of random forest for amniotic cavity organs classification

\begin{tabular}{|l|c|c|c|c|c|c|}
\hline \multirow{2}{*}{ Class } & \multicolumn{6}{|c|}{$\begin{array}{c}\text { Precision (\%) performance } \\
\text { comparison with several rectangle } \\
\text { window size }\end{array}$} \\
\cline { 2 - 7 } & $3 \times 5$ & $5 \times 3$ & $5 \times 7$ & $7 \times 5$ & $7 \times 9$ & $9 \times 7$ \\
\hline $\begin{array}{l}\text { Amniotic } \\
\text { Fluid }\end{array}$ & 98 & 96 & 100 & 99 & 98 & 88 \\
\hline Placenta & 88 & 90 & 99 & 89 & 92 & 96 \\
\hline Uterus & 100 & 84 & 99 & 89 & 86 & 100 \\
\hline $\begin{array}{l}\text { Fetal } \\
\text { Body }\end{array}$ & 90 & 90 & 100 & 100 & 98 & 84 \\
\hline $\begin{array}{l}\text { Average } \\
\text { Precision }\end{array}$ & 94 & 90 & $\mathbf{9 9 . 5}$ & 94.25 & 93.5 & 92 \\
\hline
\end{tabular}

Table 6. Recall of random forest for amniotic cavity organs classification

\begin{tabular}{|l|c|c|c|c|c|c|}
\hline \multirow{2}{*}{ Class } & \multicolumn{6}{|c|}{$\begin{array}{c}\text { Recall (\%) performance comparison } \\
\text { with several rectangle window size }\end{array}$} \\
\cline { 2 - 7 } & $3 \times 5$ & $5 \times 3$ & $5 \times 7$ & $7 \times 5$ & $7 \times 9$ & $9 \times 7$ \\
\hline $\begin{array}{l}\text { Amnioti } \\
\text { c Fluid }\end{array}$ & 98 & 92 & 99 & 99 & 95 & 87 \\
\hline Placenta & 92 & 89 & 100 & 90 & 89 & 96 \\
\hline Uterus & 99 & 88 & 100 & 88 & 89 & 97 \\
\hline $\begin{array}{l}\text { Fetal } \\
\text { Body }\end{array}$ & 86 & 98 & 99 & 100 & 100 & 87 \\
\hline $\begin{array}{l}\text { Averag } \\
\text { e Recall }\end{array}$ & $\begin{array}{c}93.7 \\
5\end{array}$ & $\begin{array}{c}91.7 \\
5\end{array}$ & $\begin{array}{c}\mathbf{9 9} \\
\mathbf{5}\end{array}$ & $\begin{array}{c}94.2 \\
5\end{array}$ & $\begin{array}{c}93.2 \\
5\end{array}$ & $\begin{array}{c}91.7 \\
5\end{array}$ \\
\hline
\end{tabular}

Table 7. Confusion matrix of random forest with $3 \times 5$ window

\begin{tabular}{|c|c|c|c|c|c|c|}
\hline \multirow{2}{*}{\multicolumn{2}{|c|}{ Cavity organs }} & \multicolumn{4}{|c|}{ Output class } & \multirow{3}{*}{$\begin{array}{l}\text { Total } \\
100\end{array}$} \\
\hline & & \multirow{2}{*}{$\begin{array}{l}\text { AF } \\
98\end{array}$} & \multirow{2}{*}{$\frac{\mathrm{PL}}{0}$} & \multirow{2}{*}{$\frac{\mathrm{UT}}{0}$} & \multirow{2}{*}{$\frac{F B}{2}$} & \\
\hline \multirow{5}{*}{ 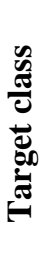 } & Amniotic & & & & & \\
\hline & Placenta & 0 & 92 & 0 & 8 & 100 \\
\hline & Uterus & 0 & 1 & 99 & 0 & 100 \\
\hline & $\begin{array}{l}\text { Fetal } \\
\text { Body }\end{array}$ & 2 & 12 & 0 & 86 & 100 \\
\hline & Total & 100 & 105 & 99 & 96 & 375 \\
\hline
\end{tabular}

Table 8 . Confusion matrix of random forest with $5 \times 3$ window

\begin{tabular}{|c|c|c|c|c|c|c|}
\hline \multirow{2}{*}{\multicolumn{2}{|c|}{ Cavity organs }} & \multicolumn{4}{|c|}{ Output class } & \multirow{3}{*}{$\begin{array}{l}\text { Total } \\
100\end{array}$} \\
\hline & & \multirow{2}{*}{$\begin{array}{l}\mathrm{AF} \\
92 \\
\end{array}$} & \multirow{2}{*}{$\begin{array}{c}\mathrm{PL} \\
0\end{array}$} & \multirow{2}{*}{$\begin{array}{c}\mathrm{UT} \\
8\end{array}$} & \multirow{2}{*}{$\begin{array}{c}\text { FB } \\
0\end{array}$} & \\
\hline \multirow{5}{*}{ 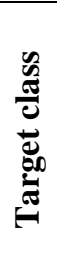 } & Amniotic & & & & & \\
\hline & Placenta & 0 & 89 & 9 & 2 & 100 \\
\hline & Uterus & 4 & 8 & 88 & 0 & 100 \\
\hline & $\begin{array}{l}\text { Fetal } \\
\text { Body }\end{array}$ & 0 & 2 & 0 & 98 & 100 \\
\hline & Total & 96 & 99 & 105 & 100 & 367 \\
\hline
\end{tabular}

99.5\% accuracy, precision, and recall. The confusion matrix random forest classification results of all window size are shown in Table 7 , $8,9,10,11$, and 12 , respectively.
Table 9. Confusion matrix of random forest with $5 \times 7$ window

\begin{tabular}{|c|c|c|c|c|c|c|}
\hline \multirow{2}{*}{\multicolumn{2}{|c|}{ Cavity organs }} & \multicolumn{4}{|c|}{ Output class } & \multirow{3}{*}{$\begin{array}{r}\text { Total } \\
100\end{array}$} \\
\hline & & \multirow{2}{*}{$\begin{array}{l}\text { AF } \\
99 \\
\end{array}$} & \multirow{2}{*}{$\begin{array}{c}\mathrm{PL} \\
0\end{array}$} & \multirow{2}{*}{$\begin{array}{c}\mathrm{UT} \\
1 \\
\end{array}$} & \multirow{2}{*}{$\begin{array}{c}\text { FB } \\
0 \\
\end{array}$} & \\
\hline \multirow{5}{*}{ 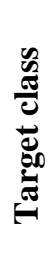 } & Amniotic & & & & & \\
\hline & Placenta & 0 & 100 & 0 & 0 & 100 \\
\hline & Uterus & 0 & 0 & 100 & 0 & 100 \\
\hline & $\begin{array}{l}\text { Fetal } \\
\text { Body }\end{array}$ & 0 & 1 & 0 & 99 & 100 \\
\hline & Total & 99 & 101 & 101 & 99 & 398 \\
\hline
\end{tabular}

Table 10. Confusion matrix of random forest with $7 \times 5$ window

\begin{tabular}{|c|c|c|c|c|c|c|}
\hline \multirow{2}{*}{\multicolumn{2}{|c|}{ Cavity organs }} & \multicolumn{4}{|c|}{ Output class } & \multirow{3}{*}{$\begin{array}{c}\text { Total } \\
100 \\
\end{array}$} \\
\hline & & \multirow{2}{*}{$\begin{array}{c}\text { AF } \\
99\end{array}$} & \multirow{2}{*}{$\begin{array}{c}\text { PL } \\
0 \\
\end{array}$} & \multirow{2}{*}{$\begin{array}{c}\mathrm{UT} \\
1\end{array}$} & \multirow{2}{*}{$\begin{array}{c}\text { FB } \\
0\end{array}$} & \\
\hline \multirow{5}{*}{ 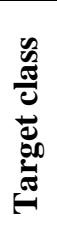 } & Amniotic & & & & & \\
\hline & Placenta & 0 & 90 & 10 & 0 & 100 \\
\hline & Uterus & 1 & 11 & 88 & 0 & 100 \\
\hline & $\begin{array}{l}\text { Fetal } \\
\text { Body }\end{array}$ & 0 & 0 & 0 & 100 & 100 \\
\hline & Total & 100 & 101 & 99 & 100 & 377 \\
\hline
\end{tabular}

Table 11. Confusion matrix of random forest with $7 \times 9$ window

\begin{tabular}{|c|c|c|c|c|c|c|}
\hline \multirow{2}{*}{\multicolumn{2}{|c|}{ Cavity organs }} & \multicolumn{4}{|c|}{ Output class } & \multirow{3}{*}{$\begin{array}{r}\text { Total } \\
100\end{array}$} \\
\hline & & \multirow{2}{*}{$\begin{array}{c}\mathrm{AF} \\
95\end{array}$} & \multirow{2}{*}{$\begin{array}{c}\mathrm{PL} \\
0\end{array}$} & \multirow{2}{*}{$\begin{array}{c}\text { UT } \\
5\end{array}$} & \multirow{2}{*}{$\begin{array}{c}\text { FB } \\
0\end{array}$} & \\
\hline \multirow{5}{*}{ 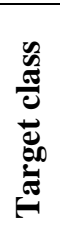 } & Amniotic & & & & & \\
\hline & Placenta & 0 & 89 & 10 & 1 & 100 \\
\hline & Uterus & 2 & 8 & 89 & 1 & 100 \\
\hline & $\begin{array}{l}\text { Fetal } \\
\text { Body }\end{array}$ & 0 & 0 & 0 & 100 & 100 \\
\hline & Total & 97 & 97 & 104 & 102 & 373 \\
\hline
\end{tabular}

Table 12. Confusion matrix of random forest with $9 \times 7$ window

\begin{tabular}{|c|c|c|c|c|c|c|}
\hline \multirow{2}{*}{\multicolumn{2}{|c|}{ Cavity organs }} & \multicolumn{4}{|c|}{ Output class } & \multirow{3}{*}{\begin{tabular}{|l} 
Total \\
100 \\
\end{tabular}} \\
\hline & & $\mathrm{AF}$ & $\mathrm{PL}$ & UT & FB & \\
\hline \multirow{5}{*}{ 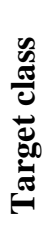 } & Amniotic & 87 & 0 & 0 & 13 & \\
\hline & Placenta & 0 & 96 & 0 & 4 & 100 \\
\hline & Uterus & 3 & 0 & 97 & 0 & 100 \\
\hline & $\begin{array}{l}\text { Fetal } \\
\text { Body }\end{array}$ & 9 & 4 & 0 & 87 & 100 \\
\hline & Total & 99 & 100 & 97 & 104 & 367 \\
\hline
\end{tabular}

*AF: Amniotic Fluid; PL: Placenta; UT: Uterus; FB: Fetal Body.

The parameters analysis of Random Forest was also carried out to determine the best value needed to achieve optimal classification results. Generally, estimator and maximum depth are important parameters in the Random Forest method used to determine the correlation between the classifier's performances. The estimator is the number of trees in the forest, while the maximum depth defines it is depth. The default values for the parameters controlling the size of the trees, such as max_depth 
and min_samples_leaf, lead to fully grown and unpruned trees that are potentially very large on some data sets. Therefore, to reduce memory consumption, the trees' complexity and size need to be controlled by setting those parameter values. Fig. 2 shows that the number of estimators or trees reached the maximum value, and it is relatively stable without changes in values above 15 . Furthermore, Fig. 3 shows that the depth of tree value reaches optimal

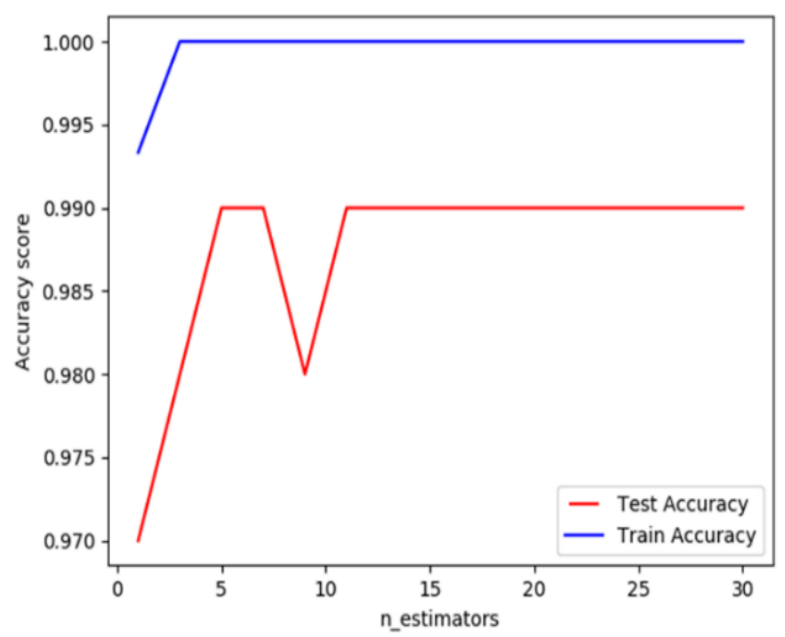

Figure. 2 Correlation plot of $n \_$estimators and accuracy of random forest with $5 \times 7$ window size

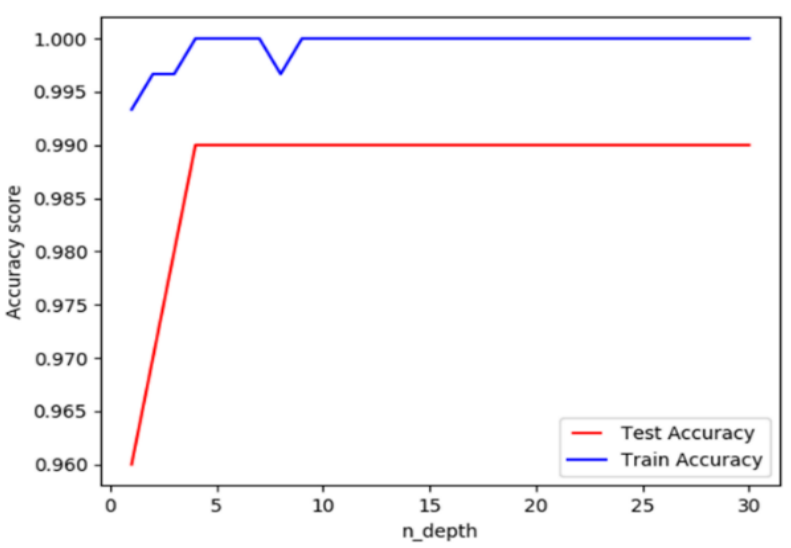

Figure. 3 Correlation plot of the $n \_$depth of tree and accuracy of random forest with $5 \times 7$ window size

Table 13. Accuracy of decision tree (C.45) for amniotic cavity organs classification

\begin{tabular}{|l|c|c|c|c|c|c|}
\hline \multirow{2}{*}{ Class } & \multicolumn{6}{|c|}{$\begin{array}{c}\text { Accuracy (\%) performance } \\
\text { comparison with several rectangle } \\
\text { window size }\end{array}$} \\
\cline { 2 - 8 } & $3 \times 5$ & $5 \times 3$ & $5 \times 7$ & $7 \times 5$ & $7 \times 9$ & $9 \times 7$ \\
\hline $\begin{array}{l}\text { Amniotic } \\
\text { Fluid }\end{array}$ & 96 & 93 & 95 & 98 & 92 & 89 \\
\hline Placenta & 91 & 86 & 98 & 83 & 89 & 94 \\
\hline Uterus & 98 & 81 & 99 & 82 & 90 & 98 \\
\hline $\begin{array}{l}\text { Fetal } \\
\text { Body }\end{array}$ & 89 & 98 & 98 & 98 & 97 & 86 \\
\hline $\begin{array}{l}\text { Average } \\
\text { Accuracy }\end{array}$ & 93.5 & 89.5 & $\mathbf{9 7 . 5}$ & 90.25 & 92 & 91.75 \\
\hline
\end{tabular}

International Journal of Intelligent Engineering and Systems, Vol.14, No.1, 2021 when it is above 10. Furthermore, performance analysis is also conducted on the decision tree method (C.45). The comparison result of the amniotic cavity organs classification with a decision tree for several size windows data accuracy, precision, and recall is shown in Tables 13, 14, and 15. Similar to the Random Forest method, the Decision Tree in the $5 \times 7$ window size is able to achieve the highest results, with $97.5 \%$ accuracy, precision, and recall, respectively.

The test results and analysis of the two different classification methods, namely Random Forest and the Decision Tree (C.45), showed that with the $5 \times 7$ window, both classifiers achieved the best results compared to other window sizes. Furthermore, the Random Forest method can achieve the best results

Table 14. Precision of decision tree (C.45) for amniotic cavity organs classification

\begin{tabular}{|l|c|c|c|c|c|c|}
\hline \multirow{2}{*}{ Class } & \multicolumn{6}{|c|}{$\begin{array}{c}\text { Precision (\%) performance comparison } \\
\text { with several rectangle window size }\end{array}$} \\
\cline { 2 - 7 } & $3 \times 5$ & $5 \times 3$ & $5 \times 7$ & $7 \times 5$ & $7 \times 9$ & $9 \times 7$ \\
\hline $\begin{array}{l}\text { Amniotic } \\
\text { Fluid }\end{array}$ & 97 & 94 & 98 & 98 & 98 & 89 \\
\hline Placenta & 90 & 85 & 95 & 82 & 90 & 94 \\
\hline Uterus & 98 & 82 & 98 & 81 & 83 & 96 \\
\hline $\begin{array}{l}\text { Fetal } \\
\text { Body }\end{array}$ & 89 & 97 & 99 & 100 & 98 & 88 \\
\hline $\begin{array}{l}\text { Average } \\
\text { Precisio } \\
\text { n }\end{array}$ & 93. & 89. & $\mathbf{9 7 .}$ & 90.2 & 92.2 & 91.7 \\
5 & 5 & $\mathbf{5}$ & 5 & 5 & 5 \\
\hline
\end{tabular}

Table 15. Recall of decision tree (C.45) for amniotic cavity organs classification

\begin{tabular}{|l|c|c|c|c|c|c|}
\hline \multirow{2}{*}{ Class } & \multicolumn{6}{|c|}{$\begin{array}{c}\text { Recall (\%) performance comparison } \\
\text { with several rectangle Window Size }\end{array}$} \\
\cline { 2 - 8 } & $3 \times 5$ & $5 \times 3$ & $5 \times 7$ & $7 \times 5$ & $7 \times 9$ & $9 \times 7$ \\
\hline $\begin{array}{l}\text { Amniotic } \\
\text { Fluid }\end{array}$ & 96 & 93 & 95 & 98 & 92 & 89 \\
\hline Placenta & 91 & 86 & 98 & 83 & 89 & 94 \\
\hline Uterus & 98 & 81 & 99 & 82 & 90 & 97 \\
\hline $\begin{array}{l}\text { Fetal } \\
\text { Body }\end{array}$ & 89 & 98 & 98 & 98 & 97 & 87 \\
\hline $\begin{array}{l}\text { Average } \\
\text { Recall }\end{array}$ & 93.5 & 89.5 & $\mathbf{9 7 . 5}$ & 90.25 & 92 & 91.75 \\
\hline
\end{tabular}

Table 16. Confusion matrix of decision tree (C.45) with $3 \times 5$ window

\begin{tabular}{|c|c|c|c|c|c|c|}
\hline \multirow{2}{*}{\multicolumn{2}{|c|}{ Cavity organs }} & \multicolumn{4}{|c|}{ Output class } & \multirow{3}{*}{$\begin{array}{l}\text { Total } \\
100\end{array}$} \\
\hline & & \multirow{2}{*}{$\begin{array}{l}\text { AF } \\
96 \\
\end{array}$} & \multirow{2}{*}{$\begin{array}{c}\text { PL } \\
0 \\
\end{array}$} & \multirow{2}{*}{$\begin{array}{c}\mathrm{UT} \\
0 \\
\end{array}$} & \multirow{2}{*}{$\frac{F B}{4}$} & \\
\hline \multirow{5}{*}{ 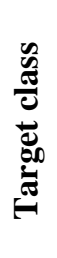 } & Amniotic & & & & & \\
\hline & Placenta & 0 & 91 & 2 & 7 & 100 \\
\hline & Uterus & 0 & 2 & 98 & 0 & 100 \\
\hline & $\begin{array}{l}\text { Fetal } \\
\text { Body }\end{array}$ & 3 & 8 & 0 & 89 & 100 \\
\hline & Total & 99 & 101 & 100 & 100 & 374 \\
\hline
\end{tabular}


Table 17. Confusion matrix of decision tree (C.45) with $5 \times 3$ window

\begin{tabular}{|c|c|c|c|c|c|c|}
\hline \multirow{2}{*}{\multicolumn{2}{|c|}{ Cavity organs }} & \multicolumn{4}{|c|}{ Output class } & \multirow{3}{*}{$\begin{array}{l}\text { Total } \\
100 \\
\end{array}$} \\
\hline & & \multirow{2}{*}{$\begin{array}{c}\text { AF } \\
93\end{array}$} & \multirow{2}{*}{$\begin{array}{c}\mathrm{PL} \\
0\end{array}$} & \multirow{2}{*}{$\frac{\mathrm{UT}}{7}$} & \multirow{2}{*}{$\begin{array}{c}\text { FB } \\
0\end{array}$} & \\
\hline \multirow{5}{*}{ 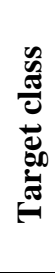 } & Amniotic & & & & & \\
\hline & Placenta & 0 & 86 & 11 & 3 & 100 \\
\hline & Uterus & 6 & 13 & 81 & 0 & 100 \\
\hline & $\begin{array}{l}\text { Fetal } \\
\text { Body }\end{array}$ & 0 & 2 & 0 & 98 & 100 \\
\hline & Total & 99 & 101 & 99 & 101 & 358 \\
\hline
\end{tabular}

Table 18. Confusion matrix of decision tree (C.45) with $7 \times 5$ window

\begin{tabular}{|c|c|c|c|c|c|c|}
\hline \multirow{2}{*}{\multicolumn{2}{|c|}{ Cavity organs }} & \multicolumn{4}{|c|}{ Output class } & \multirow[t]{2}{*}{ Total } \\
\hline & & $\mathrm{AF}$ & $\mathrm{PL}$ & UT & FB & \\
\hline \multirow{5}{*}{ 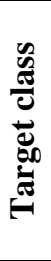 } & Amniotic & 98 & 0 & 2 & 0 & 100 \\
\hline & Placenta & 0 & 83 & 17 & 0 & 100 \\
\hline & Uterus & 2 & 16 & 82 & 0 & 100 \\
\hline & $\begin{array}{l}\text { Fetal } \\
\text { Body }\end{array}$ & 0 & 2 & 0 & 98 & 100 \\
\hline & Total & 100 & 101 & 101 & 98 & 361 \\
\hline
\end{tabular}

Table 19. Confusion matrix of decision tree (C.45) with $5 \times 7$ window

\begin{tabular}{|c|c|c|c|c|c|c|}
\hline \multirow{2}{*}{\multicolumn{2}{|c|}{ Cavity organs }} & \multicolumn{4}{|c|}{ Output class } & \multirow[t]{2}{*}{ Total } \\
\hline & & $\mathrm{AF}$ & $\mathrm{PL}$ & UT & FB & \\
\hline \multirow{5}{*}{ 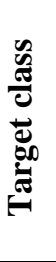 } & Amniotic & 95 & 3 & 2 & 0 & 100 \\
\hline & Placenta & 1 & 98 & 0 & 1 & 100 \\
\hline & Uterus & 1 & 0 & 99 & 0 & 100 \\
\hline & $\begin{array}{l}\text { Fetal } \\
\text { Body }\end{array}$ & 0 & 2 & 0 & 98 & 100 \\
\hline & Total & 97 & 103 & 101 & 99 & 390 \\
\hline
\end{tabular}

Table 20. Confusion matrix of decision tree (C.45) with $7 \times 9$ window

\begin{tabular}{|c|c|c|c|c|c|c|}
\hline \multirow{2}{*}{\multicolumn{2}{|c|}{ Cavity organs }} & \multicolumn{4}{|c|}{ Output class } & \multirow[t]{2}{*}{ Total } \\
\hline & & $\mathrm{AF}$ & PL & UT & FB & \\
\hline \multirow{5}{*}{ 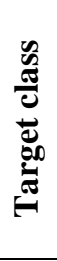 } & Amniotic & 92 & 1 & 7 & 0 & 100 \\
\hline & Placenta & 0 & 89 & 10 & 1 & 100 \\
\hline & Uterus & 2 & 7 & 90 & 1 & 100 \\
\hline & $\begin{array}{l}\text { Fetal } \\
\text { Body }\end{array}$ & 0 & 2 & 1 & 97 & 100 \\
\hline & Total & 94 & 99 & 108 & 99 & 368 \\
\hline
\end{tabular}

Table 21. Confusion matrix of decision tree (C.45) with $9 \times 7$ window

\begin{tabular}{|c|c|c|c|c|c|c|}
\hline \multirow{2}{*}{\multicolumn{2}{|c|}{ Cavity organs }} & \multicolumn{4}{|c|}{ Output class } & \multirow[t]{2}{*}{ Total } \\
\hline & & $\mathrm{AF}$ & PL & UT & FB & \\
\hline \multirow{5}{*}{ 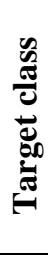 } & Amniotic & 89 & 0 & 4 & 7 & 100 \\
\hline & Placenta & 1 & 94 & 0 & 5 & 100 \\
\hline & Uterus & 2 & 0 & 98 & 0 & 100 \\
\hline & $\begin{array}{l}\text { Fetal } \\
\text { Body }\end{array}$ & 8 & 6 & 0 & 86 & 100 \\
\hline & Total & 100 & 100 & 102 & 98 & 367 \\
\hline
\end{tabular}

*AF: Amniotic Fluid; PL: Placenta; UT: Uterus; FB. Fetal Body.

Table 23. Comparison of performance previous work and proposed method for amniotic fluid segmentation

\begin{tabular}{|l|l|l|l|}
\hline Method & $\begin{array}{l}\text { Dice } \\
\text { Similarity } \\
\text { Coefficient } \\
(\%)\end{array}$ & $\begin{array}{l}\text { Jaccard } \\
\text { Coefficien } \\
\text { t/IoU (\%) }\end{array}$ & $\begin{array}{l}\text { Error } \\
\text { rate } \\
(\%)\end{array}$ \\
\hline $\begin{array}{l}\text { Encoder- } \\
\text { Decoder } \\
\begin{array}{l}\text { Convolutional } \\
\text { Network [7] }\end{array}\end{array}$ & - & 54.4 & 21.84 \\
\hline $\begin{array}{l}\text { Proposed } \\
\text { Method }\end{array}$ & $\mathbf{8 4}$ & $\mathbf{7 2 . 7}$ & $\mathbf{1 5 . 2 3}$ \\
\hline
\end{tabular}

with a performance difference of $2 \%$ for accuracy, precision, and recall values compared to the Decision Tree method (C.45).

\subsection{Segmentation result of amniotic fluid using proposed pixel classification model}

This section discusses and presents the results of the proposed pixel classification model, which is implemented to segment the amniotic fluid area on a B-mode ultrasonography image. The results were tested and evaluated using the average parameters of the dice similarity coefficient (DSC) and error rate (ER) on 50 test images. The test scenario is divided into two stages, first is testing performance model by

Table 22. Performance of proposed model in several rectangle windows

\begin{tabular}{|c|c|c|c|c|c|c|c|c|c|c|c|c|}
\hline \multirow{2}{*}{ Classifier } & \multicolumn{10}{|c|}{ Window Size } \\
\cline { 2 - 14 } & \multicolumn{2}{|c|}{$\mathbf{3 \times 5}$} & \multicolumn{2}{|c|}{$\mathbf{5 \times 3}$} & \multicolumn{2}{|c|}{$\mathbf{5 \times 7}$} & \multicolumn{2}{|c|}{$\mathbf{7 \times 5}$} & \multicolumn{2}{|c|}{$\mathbf{7 \times 9}$} & \multicolumn{2}{|c|}{$\mathbf{9 \times 7}$} \\
\cline { 2 - 13 } & DSC & $\begin{array}{c}\text { ER } \\
(\%)\end{array}$ & DSC & $\begin{array}{c}\text { ER } \\
(\%)\end{array}$ & DSC & $\begin{array}{c}\text { ER } \\
(\%)\end{array}$ & DSC & $\begin{array}{c}\text { ER } \\
(\%)\end{array}$ & DSC & $\begin{array}{c}\text { ER } \\
(\%)\end{array}$ & $\begin{array}{c}\text { DSC } \\
\begin{array}{c}\text { ER } \\
(\%)\end{array}\end{array}$ \\
\hline Random Forest & 0.73 & 36.56 & 0.82 & 15.23 & 0.76 & 19.47 & 0.84 & 18.17 & 0.71 & 21.14 & 0.73 & 23.2 \\
\hline Decision Tree & 0.66 & 46.1 & 0.80 & 16.5 & 0.76 & 19.67 & 0.80 & 22.2 & 0.83 & 17.31 & 0.70 & 30.1 \\
\hline
\end{tabular}




\section{Image test}
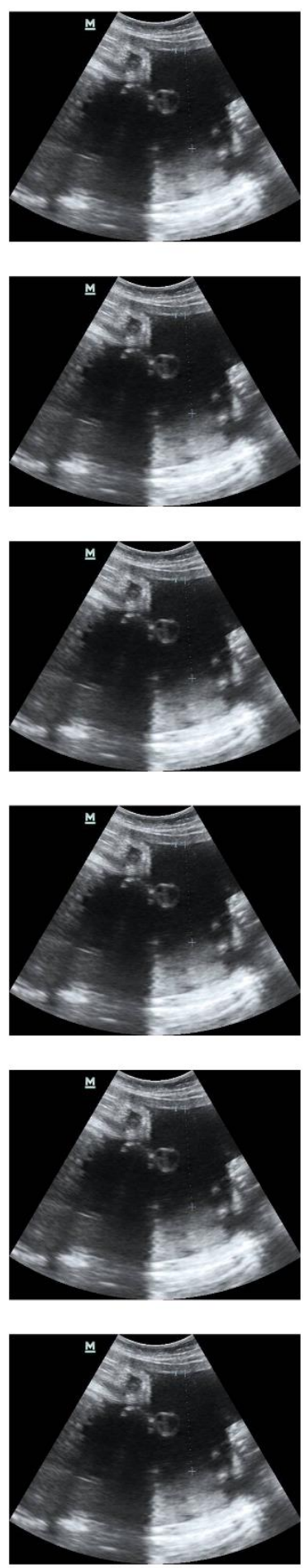

Pixel classification result
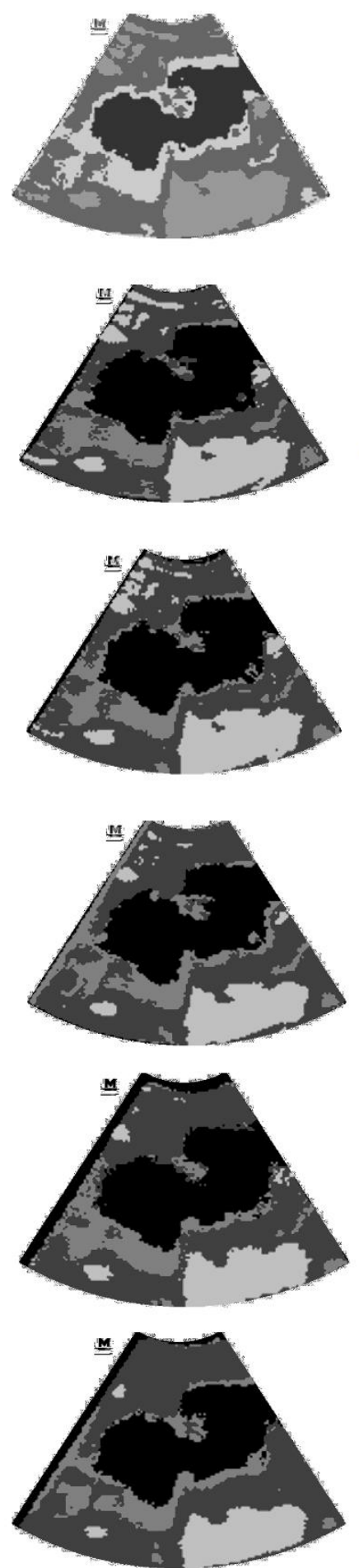

Edge detection
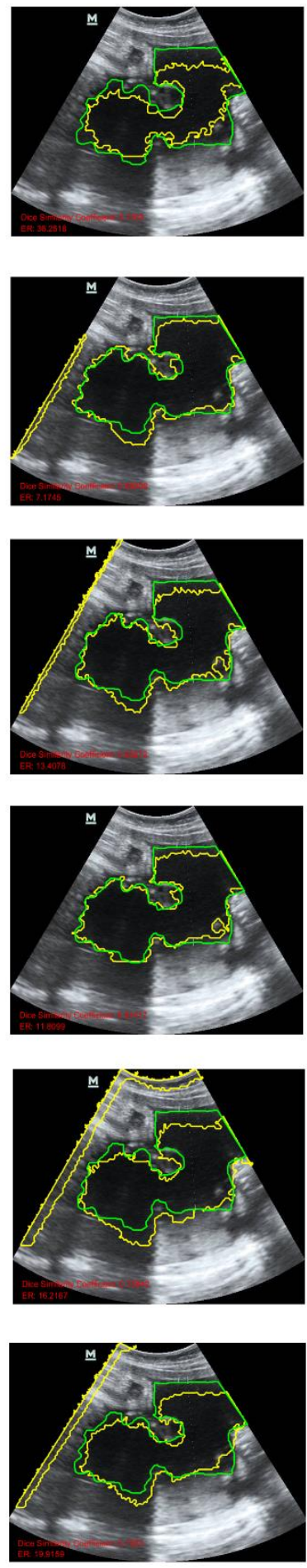

Figure. 4 Segmentation result of the proposed model using a random forest. first row: result on window size $3 \times 5$, second row: result on window size $5 \times 3$, third row: result on window size $5 \times 7$, fourth row: result on window size $7 \times 5$, fifth row: result on window size $7 \times 9$, and sixth row: result on window size $9 \times 7$

several rectangle windows and the second is comparing model against state of the art of previous model. The first test scenario is carried out for each different window size using the random forest and decision tree (C.45) methods. Furthermore, in the second scenario, the best results obtained in the first 
Image test
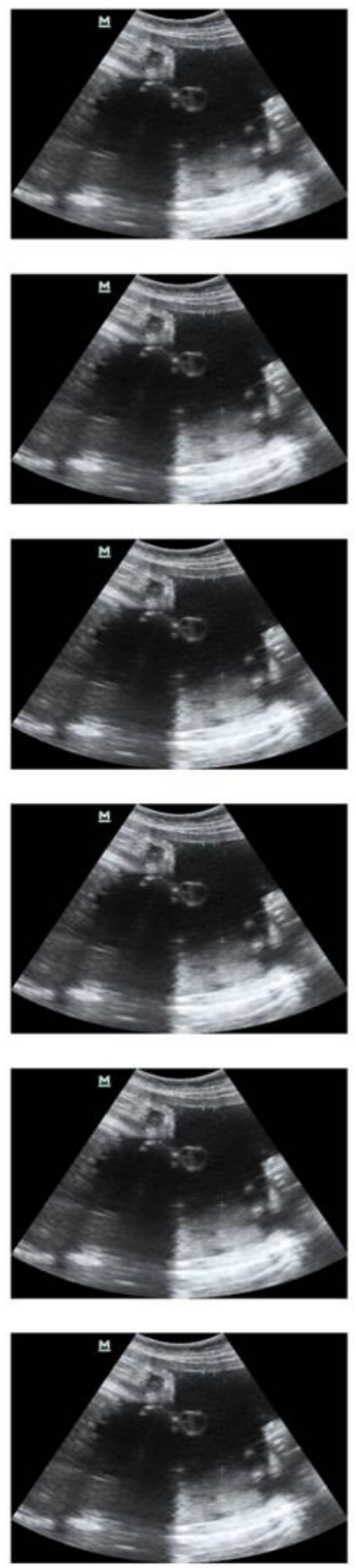

Pixel classification result
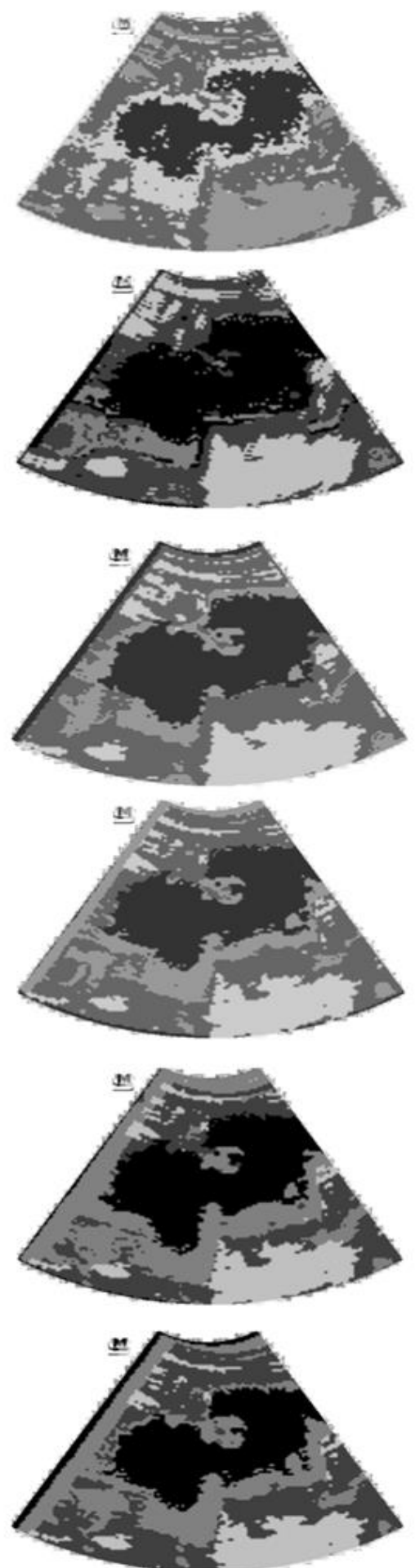

\section{Edge detection}
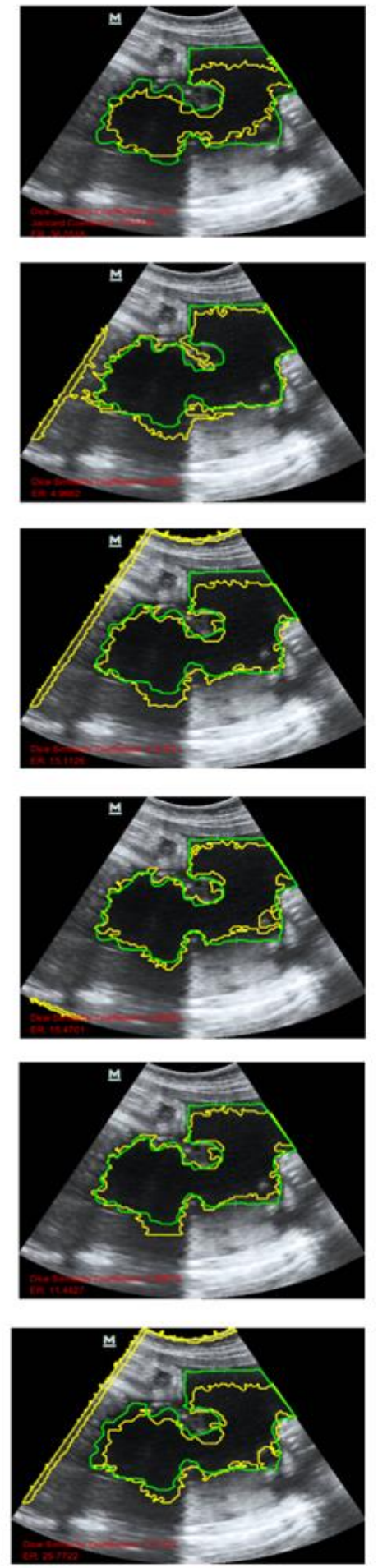

Figure. 5 Segmentation result of the proposed model using a decision tree (C.45). First row: result on window size $3 \times 5$, second row: result on window size $5 \times 3$, third row: result on window size $5 \times 7$, fourth row: result on window size $7 \times 5$, fifth row: result on window size $7 \times 9$, and sixth row: result on window size $9 \times 7$

were compared with the previous state of the art method in the research carried out by Yan lee et al.
[7]. The test results of the first scenario are shown in Table 22. 
Table 22 shows that the random forest method is able to produce the best results with an average DSC of 0.84 and an error rate of $18.17 \%$ on a window size of $7 \times 5$. Meanwhile, the decision tree method (C.45) produces the best performance on a $7 \times 9$ window with 0.83 DSC and a $17.31 \%$ Error Rate (ER). The results of segmentation and edge detection from the proposed pixel classification model using the Random Forest and Decision Tree methods with several window sizes are shown in Fig. 4 and Fig. 5. The test image is randomly taken from a collection of test images with the green line used to denote the edge of the ground truth area of the amniotic fluid, while the yellow is used to represent the proposed model. Fig. 4 and Fig. 5 shows that the new test image input, using the Random Forest method, achieves its best results in the $7 \times 5$ window with a value of 0.91 DSC and an $11.8 \%$ error rate (ER). Furthermore, in the Decision Tree method, the best result was achieved in a $7 \times 9$ window with a value of 0.89 DSC and $11.4 \%$ ER. Furthermore, the second test is carried out by comparing the results of the proposed model with the previous state of the art model. In a study conducted by Yan et al. [7]. The segmentation of amniotic fluid areas was determined using the Encoder-Decoder Convolutional Neural Network approach. The data used were 413 and 188 images for training and testing sets, respectively. The settings for USG machines used are the GE voluson E8 and C15 linear array transducer with frequencies of $4.0 \mathrm{~Hz}$ and an average fetal week of approximately 22 weeks. The comparison results of the proposed models with the state-of-the-art model from the study [7] are shown in Table 23. According to the table, the proposed model can improve performance for detecting the amniotic fluid area with an increase in the IoU/Jaccard Coefficient value by $18.3 \%$ or in other word increases by 0.183 in the $0-1$ value range.

Also, the proposed model can reduce the error rate by $6.61 \%$, which means that it can increase accuracy to $84.77 \%$. Based on the results of testing in scenarios one and two, it can be seen that the proposed model in detecting the amniotic fluid can achieves better performance from the previous model. These results are important for the continuation of research using amniotic fluid objects in the feature extraction stage and classification.

\section{Conclusion}

This research presented a pixel classification model to segment the amniotic fluid area. In the proposed model, the training set is collected based on the spatial information of each pixel on its environment within the boundaries of the rectangle window. The features used are extracted based on the pixel values in the window using the local first-order statistical method combined with the gray-level. The proposed model has the ability to provide satisfactory results, especially for amniotic fluid objects. The classification performance proposed model shows that by using Random forest with $5 \times 7$ window size can achieve the highest performance with $99.5 \%$ accuracy, precision, and recall, respectively.

Furthermore, Decision Tree is also able to achieve the highest results in the $5 \times 7$ window size, with $97.5 \%$ accuracy, precision, and recall, respectively. This shows that the window sampling model and the proposed feature are able to produce train pixel data adequately, thereby enabling the classifier method to generalize the data for each class. Furthermore, in segmenting the amniotic fluid area, the proposed method produced highest performance with a value of 0.84 DSC and $15.23 \%$ ER. It was also able to improve performance for detecting the amniotic fluid area with an increase in the IoU value by $18.3 \%$ or the Jaccard coefficient by 0.183 in the 0 1 value range compared by previous state of the art model. The proposed model also can reduce the error rate by $6.61 \%$ with an accuracy increase of $84.77 \%$. Therefore, based on these results, the proposed method can be used to feature extraction and classification stages of the amniotic fluid.

\section{Conflicts of Interest}

The authors declare there are no conflict interest in this work.

\section{Author Contributions}

Conceptualization, Desiana and Hartati; methodology, Desiana and Hartati; software, Desiana; validation, Desiana and Hartati; formal analysis, Hartati; writing - original draft preparation, Desiana and Hartati; writing - review and editing, Desiana and Hartati; supervision, Hartati; funding acquisition, Hartati”.

\section{Acknowledgments}

The authors would like to thank to the Department of Computer Science and Electronic Universitas Gadjah Mada for funding this research. The author also would thank the Surya Husada Hospital, Bali, for supporting this research in providing data.

\section{References}

[1] F. Berton, F. Cheriet, M. C. Miron, and C. Laporte, "Segmentation of the spinous process 
and its acoustic shadow in vertebral ultrasound images", Comput. Biol. Med., Vol. 72, pp.201211, 2016.

[2] C. M. A. Ten Broek, J. Bots, I. Varela-Lasheras, M. Bugiani, F. Galis, and S. Van Dongen, "Amniotic fluid deficiency and congenital abnormalities both influence fluctuating asymmetry in developing limbs of human deceased fetuses", PLoS One, Vol. 8, No.11, pp. 1-9, 2013.

[3] L. Dallaire and M. Potier, "Amniotic Fluid", Elsevier Second Edi., Vol, 2012.

[4] A. F. Nabhan and Y. A. Abdelmoula, "Amniotic fluid index versus single deepest vertical pocket: A meta-analysis of randomized controlled trials", Int. J. Gynecol. Obstet, Vol. 104, No. 3, pp. 184188, 2009.

[5] S. Q. Rashid, "Amniotic Fluid Volume Assessment Using the Single Deepest Pocket Technique in Bangladesh", J. Med. Ultrasound, Vol. 21, No. 4, pp. 202-206, 2013.

[6] K. M. Meiburger, U. R. Acharya, and F. Molinari, "Automated localization and segmentation techniques for B-mode ultrasound images: A review", Comput. Biol. Med., Vol. 92, pp. 210-235, 2018.

[7] Y. Li, R. Xu, J. Ohya, and H. Iwata, "Automatic Fetal Body and Amniotic Fluid Segmentation from Fetal Ultrasound Images by EncoderDecoder Network with Inner Layers", In: Proc. of Annu. Int. Conf. IEEE Eng. Med. Biol. Soc. EMBS, pp. 1485-1488, 2017.

[8] C. Qian and X. Yang, "An integrated method for atherosclerotic carotid plaque segmentation in ultrasound image", Comput. Methods Programs Biomed., Vol. 153, pp. 19-32, 2018.

[9] D. V. Pazinato, "Pixel-Level tissue classification for ultrasound images", IEEE J. Biomed. Heal. Informatics, Vol. 20, No. 1, pp. 256-267, 2016.

[10] S. Rosati, K. M. Meiburger, G. Balestra, U. R. Acharya, and F. Molinari, "Carotid Wall Measurement and Assessment Based on PixelBased and Local Texture Descriptors" J. Mech. Med. Biol, Vol. 16, No. 01, pp. 1640006, 2016.

[11] S. Yin, "Automatic kidney segmentation in ultrasound images using subsequent boundary distance regression and pixelwise classification networks", Med. Image Anal, Vol. 60, pp. 101602, 2020.

[12] F. Berton, W. Azzabi, F. Cheriet, and C. Laporte, "Automatic segmentation of vertebrae in ultrasound images", Comput. Sci. Artif. Intell. Lect. Notes Bioinformatics, Vol. 9164, pp. 344351, 2015.
[13] F. Chen, R. Ma, J. Liu, M. Zhu, and H. Liao, "Lumen and media-adventitia border detection in IVUS images using texture enhanced deformable model", Comput. Med. Imaging Graph, Vol. 66, No. pp. 1-13, 2018.

[14] K. R. Gray, P. Aljabar, R. A. Heckemann, A. Hammers, and D. Rueckert, "Random forestbased similarity measures for multi-modal classification of Alzheimer's disease", Neuroimage, Vol. 65, pp. 167-175, 2013.

[15] B. C. Ko, S. H. Kim, and J. Y. Nam, "X-ray image classification using random forests with local wavelet-based CS-local binary patterns", $J$. Digit. Imaging, Vol. 24, No. 6, pp. 1141-1151, 2011.

[16] P. Poudel, A. Illanes, E. J. G. Ataide, N. Esmaeili, S. Balakrishnan, and M. Friebe, "Thyroid Ultrasound Texture Classification Using Autoregressive Features in Conjunction with Machine Learning Approaches", IEEE Access, Vol. 7, pp. 79354-79365, 2019.

[17] P. D. W. Ayu, S. Hartati, and A. Musdholifah, "Amniotic Fluid Segmentation by Pixel Classification in B-Mode Ultrasound Image for Computer Assisted Diagnosis", In: Proc. of Int. Conf. Soft Comput. Data Sci, Singapore, pp. 59 70, 2019.

[18] X. Y. Wang, Z. F. Wu, L. Chen, H. L. Zheng, and H. Y. Yang, "Pixel classification based color image segmentation using quaternion exponent moments", Neural Networks, Vol. 74, pp. 1-13, 2016.

[19] D. A. Tyas, S. Hartati, A. Harjoko, and T. Ratnaningsih, "Morphological , Texture , and Color Feature Analysis for Erythrocyte Classification in Thalassemia Cases", IEEE Access, Vol. 8, pp. 69849-69860, 2020.

[20] S. Hartati, A. Harjoko, R. Rosnelly, and I. Chandradewi, "Performance of SVM and ANFIS for Classification of Malaria Parasite and Its Life-Cycle-Stages in Blood Smear", In: Proc. of Soft Comput. Data Sci, CCIS 937, pp. 110 121, 2019. 